\title{
An integrated optimization algorithm for parameter structure identification in groundwater modeling
}

\author{
Chung-Che Tan ${ }^{\mathrm{a}}$, Ching-Pin Tung ${ }^{\mathrm{a}}$, Chu-Hui Chen ${ }^{\mathrm{b}}$, William W.-G. Yeh ${ }^{\mathrm{c}, *}$ \\ ${ }^{a}$ Department of Bioenvironmental Systems Engineering, National Taiwan University, No. 1, Sec. 4, Roosevelt Road, Taipei, Taiwan \\ ${ }^{\mathrm{b}}$ Department of Civil Engineering, China University of Technology, No. 56, Sec. 3, Shinglung Road, Taipei, Taiwan \\ ${ }^{\mathrm{c}}$ Department of Civil and Environmental Engineering, UCLA, 5731 Boelter Hall, Los Angeles, CA 90095, United States
}

Received 20 August 2007; received in revised form 28 November 2007; accepted 29 November 2007

Available online 14 December 2007

\begin{abstract}
The inverse problem of parameter structure identification in a distributed parameter system remains challenging. Identifying a more complex parameter structure requires more data. There is also the problem of over-parameterization. In this study, we propose a modified Tabu search for parameter structure identification. We embed an adjoint state procedure in the search process to improve the efficiency of the Tabu search. We use Voronoi tessellation for automatic parameterization to reduce the dimension of the distributed parameter. Additionally, a coarse-fine grid technique is applied to further improve the effectiveness and efficiency of the proposed methodology. To avoid over-parameterization, at each level of parameter complexity we calculate the residual error for parameter fitting, the parameter uncertainty error and a modified Akaike Information Criterion. To demonstrate the proposed methodology, we conduct numerical experiments with synthetic data that simulate both discrete hydraulic conductivity zones and a continuous hydraulic conductivity distribution. Our results indicate that the Tabu search allied with the adjoint state method significantly improves computational efficiency and effectiveness in solving the inverse problem of parameter structure identification.
\end{abstract}

(C) 2007 Elsevier Ltd. All rights reserved.

Keywords: Parameter structure identification; Tabu search; Adjoint state method; Voronoi tessellation; Parameter uncertainty; Akaike information criterion

\section{Introduction}

Distributed parameter models are used to simulate groundwater flow, but the reliability of results is strongly dependent on whether the model parameters are properly identified. However, the real geological structure of an aquifer is generally very complex and mostly unknown. More data are required for identifying a more complex parameter structure, and there is always the problem of over-parameterization. Additionally, the inverse problem of parameter structure identification is highly non-linear

\footnotetext{
* Corresponding author. Tel.: +1 3108252300 ; fax: +13108257581.

E-mail addresses: d92622008@ntu.edu.tw (C.-C. Tan), cptung@ntu. edu.tw (C.-P. Tung), yawei@cute.edu.tw (C.-H. Chen), williamy@seas. ucla.edu (W.W.-G. Yeh).
}

and non-convex; hence, gradient-based algorithms often get trapped in local optima. On the other hand, heuristic search algorithms usually require a very large number of simulation runs. There remains a need to develop efficient inverse solution algorithms.

Although the inverse problem in groundwater modeling has been studied for over four decades, identifying the spatial distribution of a heterogeneous aquifer remains formidable because of the limitation in both quantity and quality of data. The inverse problem is inherently ill-posed in that the solution is usually non-unique or unstable. As pointed out by Yeh [1], parameterization is always necessary in order to reduce the parameter dimension of a distributed parameter to a finite dimensional form so that a stable and unique solution of the inverse problem can be sought. 
Parameterization as well as the efficiency and effectiveness of the inverse algorithm are the two most important components in parameter structure identification. In this study, Tabu search is allied with the adjoint state method to improve the search efficiency and parameterization is achieved by Voronoi tessellation (VT).

Sykes et al. [2] developed adjoint sensitivity theory for both the continuous and discrete (numerical) equations of two-dimensional steady state flow in a confined aquifer. They derived a performance measure with respect to the system parameters, such as the piezometric heads, velocities, travel time, and mass discharge with respect to recharge-discharge rates, prescribed boundary heads or fluxes, thicknesses, and hydraulic conductivities. As Yeh [1] pointed out, there are basically three methods, the influence coefficient method, the sensitivity equation method, and the variational or adjoint state method (ASM) for calculating the Jacobian matrix (parameter sensitivity matrix). The ASM is particularly efficient when the sensitivities of a large number of parameters are required. Sun and Yeh [3] proposed a general procedure for deriving the adjoint state equations and described their associated conditions for solving coupled inverse problems in groundwater modeling.

In recent years, heuristic algorithms have been used to identify model parameters in groundwater modeling. Zheng and Wang [4] used Tabu search (TS) and simulated annealing (SA) to search for an optimal hydraulic conductivity zonation structure in one-dimensional problems. Tsai et al. [5] developed a sequential global-local optimization method, which consists of a genetic algorithm (GA), a quasi-Newton method, and local search to solve the combinatorial problem. More recently, Tung and Chou [6] and Tung and Tan [7] applied heuristic optimization algorithms, such as TS and SA, with different zonation methods to identify the spatial distribution of hydraulic conductivity or transmissivity. This study proposes to use the ASM to improve computational efficiency of TS. We note that TS requires running the simulation model $N$ times to evaluate $N$ neighbor solutions. More neighbor solutions require more simulation runs. When TS is allied with the ASM, it only requires running the simulation model twice regardless of how many neighbor solutions there are. Details will be explained in a later section.

To reduce the parameter dimension of a distributed parameter to a finite dimensional form, either zonation or interpolation can be used [1]. Doherty [8] used the pilot point method as an interpolation method to identify the spatial distribution of transmissivity. Tsai et al. [5] and Tung and Tan [7] applied Voronoi tessellation (VT) to automatically parameterize the zonation pattern of hydraulic conductivity or transmissivity. Tung and Chou [6] proposed a pattern zonation based on the pattern classification to identify the spatial distribution of pumping.

In this paper, we first use VT to automatically parameterize the distributed hydraulic conductivity for a given parameter dimension, represented by the number of basis points. Second, the ASM is used to improve the efficiency of the TS search. To further improve computational efficiency, we apply a coarse-fine grid search over the modified TS. For each parameter dimension, we calculate the fitting residual error, the parameter uncertainty error and the modified Akaike Information Criterion. We use these three indices to determine the optimal level of parameter complexity. Numerical experiments are carried out to demonstrate the validity and computational efficiency of the proposed approach.

The rest of this paper is organized as follows. In Section 2 , the inverse problem and its formulation for parameter structure identification in groundwater modeling are introduced. The stopping criteria used to determine the optimal level of parameter complexity are also described. The proposed integrated optimization algorithm, which consists of VT, TS and the ASM are illustrated in Section 3. Section 4 outlines the numerical experiments with hypothetical study fields and the proposed integrated optimization algorithm is applied with the coarse-fine grid search technique. We then summarize the results with conclusions.

\section{Inverse problem of groundwater modeling}

Sun et al. [9] proposed a procedure for parameter structure identification that starts from a simpler parameter structure and gradually increases the parameter dimension by adding one zone at a time until the stopping criteria are satisfied. At each level of parameter complexity, the fitting residual error and the model structure error of using a simplified model to replace a more complex model were computed. In this study, we employ the same concept of systematically increasing parameter complexity in order to capture the spatial variation of the hydraulic conductivity. However, a key purpose of our study is to develop a computationally more effective and efficient integrated inverse algorithm.

\subsection{Groundwater flow equations}

Eqs. (1)-(3) show the governing equation of a twodimensional, transient flow for a heterogeneous, isotropic and confined aquifer as well as the associated initial and boundary conditions.

$$
\begin{aligned}
& S \frac{\partial \phi}{\partial t}-\nabla \cdot(T \nabla \phi)-Q \delta\left(x-x_{i}\right) \delta\left(y-y_{i}\right)=0, \\
& \quad(x, y) \in(\Omega), \quad 0 \leqslant t \leqslant t_{f}, \\
& \text { initial condition : } \phi(x, y, 0)=f_{0}(x, y), \quad(x, y) \in(\Omega), \\
& \text { boundary condition }:\left.\phi\right|_{\left(\Gamma_{1}\right)}=f_{1}(t),\left.\quad T \nabla \phi \cdot n\right|_{\left(\Gamma_{2}\right)} \\
& =f_{2}(t) \quad 0 \leqslant t \leqslant t_{f},
\end{aligned}
$$

where $\phi(x, y, t)$ is the head $[L] ; S(x, y)$ is the storage coefficient (dimensionless) which is the specific storage $[1 / L]$ multiplied by the aquifer thickness $[L] ; T(x, y)$ is the transmissivity $\left[L^{2} / T\right] ; Q(x, y, t)$ is the source/sink term $\left[L^{3} / T\right]$; 
$\delta$ is the Dirac function $[1 / L] ; x$ and $y$ are the space variables $[L] ; t$ is the time variable $[T] ;(\Omega)$ is the flow region; $\Gamma_{1}$ and $\Gamma_{2}$ are the boundary of the aquifer $\left(\Gamma=\Gamma_{1} \cup \Gamma_{2}\right) ; n$ is the normal derivative of $\Gamma_{2} ; f_{0}, f_{1}, f_{2}$ are specified functions; and $t_{f}$ is the simulation ending time. With known parameters $S, T$ and $Q$, Eq. (1) is solved to estimate the heads $\phi$ with various numerical methods and software packages, such as MODFLOW [10]. This is a procedure for solving the forward problem. However, a successful prediction of head $\phi$ requires first solving the inverse problem to identify the parameters imbedded in the governing equation.

\subsection{Objective function of the inverse problem}

To illustrate the proposed integrated optimization algorithm, we assume that transmissivity (or hydraulic conductivity) is the distributed parameter to be identified from head observations and that all other parameters in Eq. (1) are known. Generally, the performance criterion used to solve the inverse problem is the residual error between observed and simulated heads. Thus, the residual error as well as the objective function $E(T)$ is

$\operatorname{Min}_{T(x, y)} E(T)=\left\|\phi^{\operatorname{sim}}(T)-\phi^{\text {obs }}\right\|, \quad T(x, y) \in \Omega_{T}$,

where $\phi^{\text {sim }}$ is the vector of the simulated heads at the observation locations using an estimated parameter structure $T(x, y) ; \phi^{\text {obs }}$ is the vector of observed heads; $\Omega_{T}$ is the admissible set of $T(x, y)$; and $\|\cdot\|$ is a norm. From Eq. (4), however, transmissivity can be regarded as the hydraulic conductivity because a constant thickness is assumed for the aquifer.

\subsection{Stopping criteria for parameter structure identification}

How to determine the optimal number of zones is a critical issue in parameter identification. An increase in parameter dimension by increasing the number of zones will decrease the residual error. However, at some point an increase in parameter dimension will result in a drastic increase in parameter uncertainty error and over-parameterization occurs. Yeh and Yoon [11] and Tsai et al. [5] studied the tradeoff between the residual error and the parameter uncertainty error. Other than the parameter uncertainty error, several model selection criteria based on information theory have been developed for determining model complexity, such as the Akaike Information Criterion $(A I C)$ [12] and the modified $A I C$ for small samples $\left(A I C_{C}\right)[13]$. The preferred model is the one with the smallest value of $A I C$ or $A I C_{c}$. In this study, the parameter uncertainty and $A I C_{c}$ are used as the criteria to determine the optimal parameter dimension.

We adopt a covariance matrix to quantify the parameter uncertainty and the trace or the norm of the covariance matrix can be used to compute the overall parameter uncertainty at $n$ computation nodes. The covariance matrix of an estimated parameter can be expressed as $[11,7]$
$\operatorname{Cov}_{n}\left(\widehat{T}_{n}\right)=G_{m}\left(\frac{J\left(\widehat{T}_{m}\right)}{N_{\mathrm{o}}-m}\left[A\left(\widehat{T}_{m}\right)\right]^{-1}\right) G_{m}^{\mathrm{T}}$,

where $G_{m}$ is the structure matrix, $J\left(\widehat{T}_{m}\right)$ is the residual error, $N_{\mathrm{o}}$ is the number of observations, $m$ is the number of parameters, $A$ is $\left[J_{D}^{\mathrm{T}} J_{D}\right]$, and $J_{D}$ is Jacobian matrix of heads with respect to parameter structure $\widehat{T}_{m}$. A norm of the covariance matrix, such as the trace, can be used to represent overall parameter uncertainty.

Hurvich and Tsai [13] obtained a bias-corrected version of the Akaike Information Criterion, called $A I C_{c}$, and extended the applicability of $A I C_{c}$ to both regression and time series. $A I C_{c}$ is the sum of $A I C$ and an additional non-stochastic penalty term. An equivalent form is

$A I C_{c}=A I C+\frac{2(m+1)(m+2)}{N_{\mathrm{o}}-m-2}$,

where

$A I C=N_{\mathrm{o}} \log \left(\frac{J(\widehat{T})}{N_{\mathrm{o}}}\right)+2(m+1)$.

In Eqs. (6) and (7), $J(\widehat{T})$ is the residual error, $N_{\mathrm{o}}$ is the number of observations, and $m$ is the number of parameters. The suggested model parameter structure should have the smallest value of $A C I_{c}$. The minimized $A C I_{c}$ seeks a compromise between model simplicity and complexity.

\section{An integrated optimization algorithm}

In the proposed integrated optimization algorithm, for a given parameter dimension (number of zones) the spatial distribution of hydraulic conductivity is first parameterized by VT. Then, TS allied with the ASM is used to optimize the basis point locations and their associated values. We further use a coarse-fine grid technique to improve the solution. The manner in which these methods are combined is described below.

\subsection{Voronoi tessellation (VT)}

As with a distributed parameter model, it is unrealistic to determine the parameter value for each grid associated with the numerical model. Parameterization is necessary in order to reduce the parameter dimension. The zonation and interpolation methods are two different popular means of parameterization [1]. VT is an automatic zonation method that tessellates the domain into a set of Voronoi polygons given a set of distinct points in space [5]. VT was first introduced by mathematicians Dirichlet [14] and Voronoi [15], and has been applied to many fields. More recently, Tsai et al. [5] and Tung and Tan [7] have successfully applied VT to identify the equivalent zone-based hydraulic conductivity for a heterogeneous field. Although VT has been studied for more than 150 years, it remains a powerful tool for spatial and pattern analysis [16].

To construct VT, a finite number of points, $m$, and their location in a Euclidean plane are first given and 
$2 \leqslant m<\infty$. The $m$ basis points are respectively labeled by $p_{1}, \ldots, p_{m}$ with locations $\left(p x_{1}, p y_{1}\right), \ldots,\left(p x_{m}, p y_{m}\right)$ and the associated parameter values are $P V\left(p x_{i}, p y_{i}\right)$. Let $P V^{e}(p x, p y)$ be an estimated parameter value in the Euclidean plane with location $p^{e}(p x, p y)$ over $n$ computation nodes. The Euclidean distance from $p^{e}$ to $p_{i}$ is $\left\|p^{e}-p_{i}\right\|=$ $\sqrt{\left(p x-p x_{i}\right)^{2}+\left(p y-p y_{i}\right)^{2}}$. If $p_{i}$ is the nearest point from $p^{e}, p^{e}$ is assigned to the zone $Z_{i}^{\mathrm{VT}}$ containing the basis point $p_{i}$ with the value $P V\left(p x_{i}, p y_{i}\right)$. Each zone $Z_{i}^{\mathrm{VT}}$ can be represented as

$Z_{i}^{\mathrm{VT}}=\left\{p^{e}\left\|p^{e}-p_{i}\right\| \leqslant\left\|p^{e}-p_{j}\right\|\right.$ for $\left.i \neq j, j \in I_{n}\right\}$,

where $I_{n}$ is an integer set. Meanwhile, each zone $Z_{i}^{\mathrm{VT}}$ has a uniform parameter value $P V\left(p x_{i}, p y_{i}\right)$. Accordingly, a plane $Z^{\mathrm{VT}}$ can be divided into a set of homogeneous zones and is given as

$Z^{\mathrm{VT}}=\left\{Z_{1}^{\mathrm{VT}}\left(p_{1}\right), \ldots, Z_{n}^{\mathrm{VT}}\left(p_{n}\right)\right\}$,

where $p_{i}$ is the basis point of zone $Z_{i}^{\mathrm{VT}}$, and the set $\left\{p_{1}, \ldots, p_{m}\right\}$ is called the basis set. Fig. 1 shows an example in which the zonation of hydraulic conductivity is represented by three basis points with coordinates $(2,4),(3,9)$, and $(7,10)$. For the rigorous mathematical properties and applications of VT, readers are referred to Okabe et al. [16]. According to VT, there are three parameters for each zone: $p x_{i}, p y_{i}$, and $P V\left(p x_{i}, p y_{i}\right)$. Increasing one zone requires three more parameters.

\subsection{Tabu search (TS)}

Fig. 2 shows the flowchart of TS. TS explores the neighbor solutions of a current solution and moves to the best one in the neighborhood. The process updates the best solution recorded once the current solution is superior and continues until the stopping criteria are met. Furthermore, TS has the ability to prevent the search from being trapped in a local optimum. This core ability of TS results from the constraints of a Tabu list which memorizes the search history. Besides the Tabu list, neighborhood and movement, the aspiration criteria and the principle to stop searching are also important components of TS. These components are briefly introduced in this section. More detailed descriptions of TS can be found in Glover and Laguna [17].

\subsubsection{Neighborhood and movement}

All the parameters of a distributed numerical model are discretized. The solution space of the parameters depends on the number of parameters and their discretization. In the process of parameter identification, any one of the parameters of the current solution being modified by a $\Delta$ (one step size) is defined as a neighbor solution, and all neighbor solutions form a neighborhood of a current solution. Moving one step from a current solution to its neighbor solution is called a movement. A more complex parameter structure has more parameters and thus results in a larger solution space and larger neighborhood. Each iteration in TS moves to the best neighbor solution and is not tabued. The best neighbor solution is then used as a new current solution for the next iteration. If there are $N$ variables and each variable can be modified by $+\Delta$ or $-\Delta$, there are $(2 \times N)$ neighbor solutions. Therefore, TS requires evaluating $(2 \times N)$ neighbor solutions, i.e. running the simulation model $(2 \times N)$ times, before taking a movement. More neighbor solutions require more computations

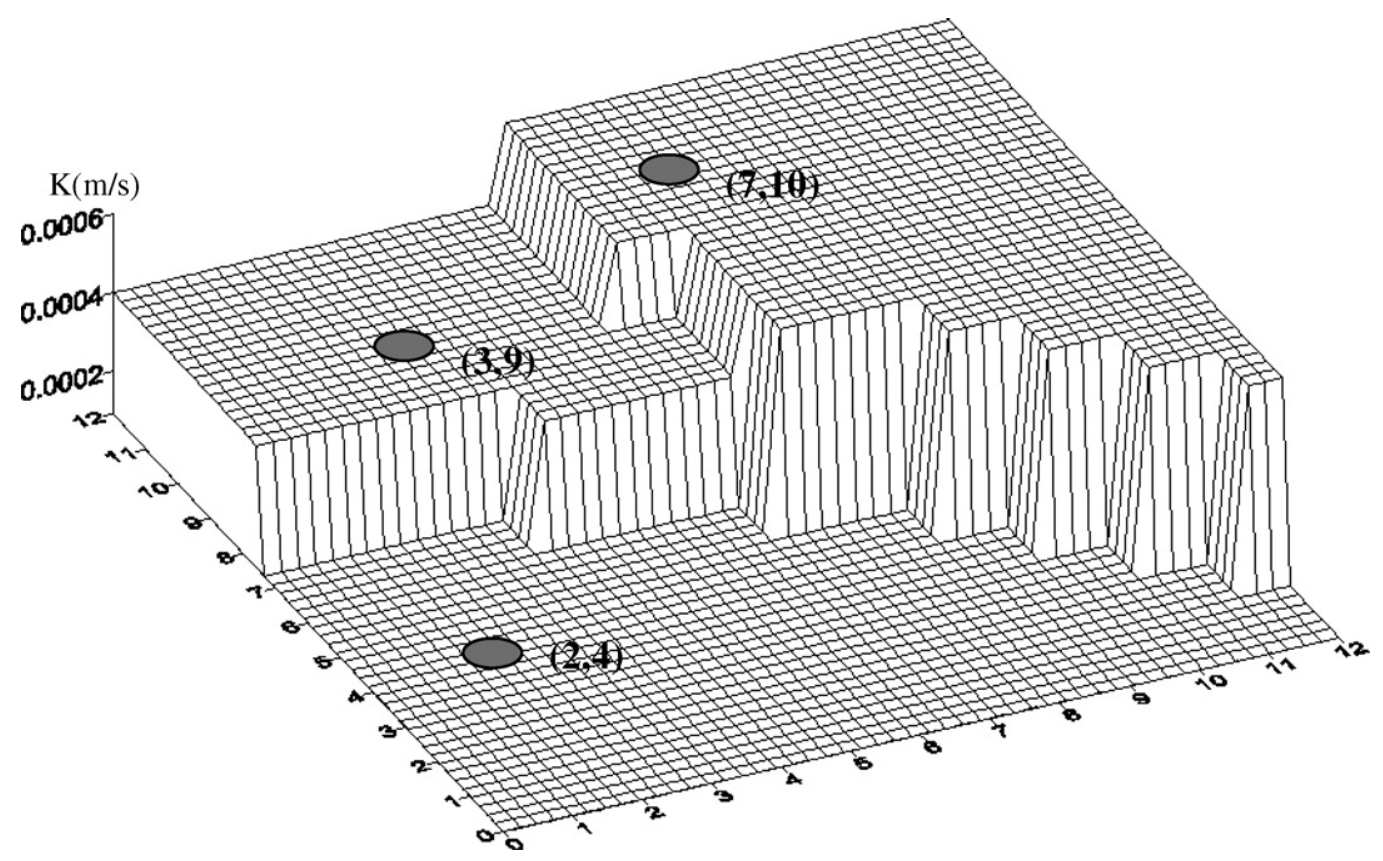

Fig. 1. The spatial distribution of hydraulic conductivity of Voronoi tessellation (VT) $(X$ and $Y$ axes represent the study area with $12 \times 12$ grids). 


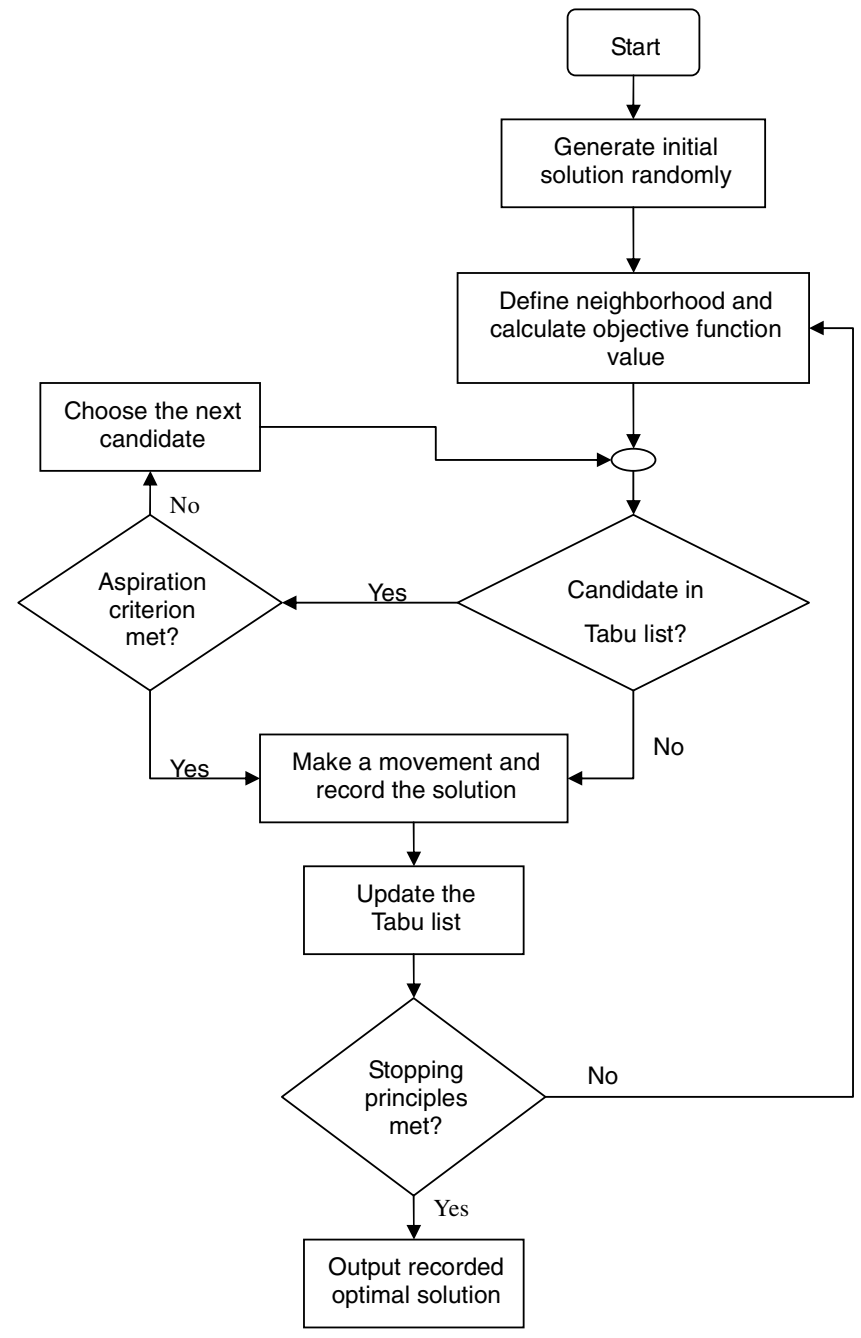

Fig. 2. Tabu search flowchart.

to determine a movement. Furthermore, more movements may be required to explore a larger solution space to find the optimum. In this study, the ASM is applied to enhance the efficiency of movement by reducing the computation from $(2 \times N)$ times to twice regardless of how large the number $N$ is.

\subsubsection{Tabu list}

The Tabu list serves as a memory to direct or prohibit movements. There are four types of memory: recency, frequency, quality, and influence [17]. The recency memory records recently explored current solutions and prohibits the search process from moving back to these solutions to avoid being trapped in a local optimum. This type of memory is adopted in this study. Moreover, the length of the Tabu list should be sufficiently long to avoid falling in a search cycle. On the other hand, the length of the Tabu list cannot be too long, because it may limit the search space to a small feasible region and could reduce the search efficiency. There is no universal principle for determining the length of the Tabu list. The length can be an arbitrarily small number as an initial guess. Then, the length of the Tabu list can be increased, if necessary, to avoid cycling.

\subsubsection{Aspiration criterion}

The Tabu list can be designed to memorize the entire current solutions recently explored or memorize parts of the variables of a solution. If the Tabu list only records the modified parameters of current solutions, it may be too rigid and require aspiration criteria. For example, although the value of some parameter of a neighbor solution is tabued, if the neighbor solution has a better objective function value than the recorded best solution, it is a never-visited solution and should not be tabued. Then, an aspiration criterion is applied to keep TS moving forward, i.e., omitting the Tabu. There are some other different aspiration criteria associated with different definitions of how to record the solutions [6]. However, all aspiration criteria have the same purpose - to ensure that the search process continues to move forward until a stopping criterion is reached.

\subsubsection{Principles for stopping the search process}

There are different principles for stopping TS, including (1) the current solution satisfies the pre-defined residual error tolerance; (2) the given number of maximum iterations is reached; and (3) successive iterations do not improve the objective function. The most adequate selection of the stopping criterion is case-dependent. Additionally, after each movement, the current solution is compared to the best-recorded solution which can be replaced by any better current solution. When the search process is stopped, the final best-recorded solution is the selected optimal solution.

\subsection{Adjoint state method (ASM)}

The ASM based on the variational theory has been used in groundwater modeling for more than three decades [18]. The most common application of the ASM in environmental applications is for improving the computational efficiency of sensitivity analyses [19]. In this study, the ASM is applied to derive the adjoint equation of the groundwater governing equation

$$
\begin{gathered}
\int_{0}^{t_{f}} \int_{(\Omega)}\left[-S \frac{\partial \psi}{\partial t}-\nabla \cdot(T \nabla \psi)\right] \delta \phi \cdot \mathrm{d} \Omega \mathrm{d} t \\
+\int_{0}^{t_{f}} \int_{(\Omega)}(\nabla \psi \cdot \nabla \phi) \delta T \cdot \mathrm{d} \Omega \mathrm{d} t=0,
\end{gathered}
$$

where $\psi(x, y, t)$ is an arbitrary function having continuous second-order space derivatives on $(\Omega)$ and first-order time derivative in $\left[0, t_{f}\right]$.

The adjoint equation is used to calculate the sensitivity of the objective function to direct TS's search process. A detailed derivation of the adjoint equation can be found in Sun [18]. Meanwhile, in order to estimate transmissivity 
$T(x, y)$ of the inverse problem of Eq. (4), an objective function $E(\cdot)$ is revised to measure the residual error of the simulated and observed heads. Moreover, the objective function $E(\cdot)$ is the integration of the performance function $f(\cdot)$ through time and space:

$$
\begin{aligned}
f[\phi( & \left.x, y, t),\left(p x_{1}, p y_{1}, T_{1}\right),\left(p x_{2}, p y_{2}, T_{2}\right), \ldots,\left(p x_{m}, p y_{m}, T_{m}\right)\right] \\
= & \sum_{i=1}^{I} \sum_{j=1}^{J}\left\{\phi ^ { \mathrm { sim } } \left[(x, y, t),\left(p x_{1}, p y_{1}, T_{1}\right),\left(p x_{2}, p y_{2}, T_{2}\right)\right.\right. \\
& \left.\left.\quad \ldots,\left(p x_{m}, p y_{m}, T_{m}\right)\right]-\phi^{\mathrm{obs}}(x, y, t)\right\}^{2} \\
& \times \delta\left(x-x_{i}^{\mathrm{obs}}\right) \delta\left(y-y_{i}^{\mathrm{obs}}\right) \delta\left(t-t_{j}^{\mathrm{obs}}\right)
\end{aligned}
$$

and

$$
\begin{aligned}
& E\left[\phi(x, y, t),\left(p x_{1}, p y_{1}, T_{1}\right),\left(p x_{2}, p y_{2}, T_{2}\right), \ldots,\left(p x_{m}, p y_{m}, T_{m}\right)\right] \\
& =\int_{0}^{t_{f}} \int_{\Omega} f\left(\phi(x, y, t),\left(p x_{1}, p y_{1}, T_{1}\right),\left(p x_{2}, p y_{2}, T_{2}\right), \ldots,\right. \\
& \left.\quad\left(p x_{m}, p y_{m}, T_{m}\right)\right) \mathrm{d} \Omega \mathrm{d} t,
\end{aligned}
$$

where $p x_{m}$ and $p y_{m}$ are the variables of VT with the $m$ th zone and $T_{m}$ represents $P V\left(p x_{m}, p y_{m}\right)$. The term $\delta(\cdot)$ represents the Dirac function; $\left(x_{i}, y_{i}\right)(i=1,2, \ldots, I)$ are locations of observations wells; and $t_{j}(j=1,2, \ldots, J)$ are the observation times.

From Eq. (12), any variation $\delta p x_{m}, \delta p y_{m}$, and $\delta T_{m}$ will cause a variation of $E(\cdot)$. Taking a variation of $E(\cdot)$ yields

$\delta E=\int_{0}^{t_{f}} \int_{(\Omega)}\left(\frac{\partial f}{\partial \phi} \delta \phi+\frac{\partial f}{\partial p x_{m}} \delta p x_{m}+\frac{\partial f}{\partial p y_{m}} \delta p y_{m}+\frac{\partial f}{\partial T_{m}} \delta T_{m}\right) \mathrm{d} \Omega \mathrm{d} t$.

Furthermore, $\delta T$ can be represented as

$\delta T=\frac{\partial T}{\partial p x_{m}} \delta p x_{m}+\frac{\partial T}{\partial p y_{m}} \delta p y_{m}+\frac{\partial T}{\partial T_{m}} \delta T_{m}$.

The terms $\delta E / \delta p x_{m}, \delta E / \delta p y_{m}$, and $\delta E / \delta T_{m}$ now can be derived, respectively, with Eqs. (10), (13), and (14). For example, to derive $\delta E / \delta p x_{m}$, Eqs. (10) and (14) can be used to obtain

$$
\begin{gathered}
\int_{0}^{t_{f}} \int_{(\Omega)}\left[-S \frac{\partial \psi}{\partial t}-(T \nabla \psi)\right] \delta \phi \mathrm{d} \Omega \mathrm{d} t+\int_{0}^{t_{f}} \int_{(\Omega)}(\nabla \psi \nabla \phi) \\
\times\left(\frac{\partial T}{\partial p x_{m}} \delta p x_{m}+\frac{\partial T}{\partial p y_{m}} \delta p y_{m}+\frac{\partial T}{\partial T_{m}} \delta T_{m}\right) \mathrm{d} \Omega \mathrm{d} t=0
\end{gathered}
$$

Since the left hand side of Eq. (15) is equal to zero, it can be added to Eq. (13), yielding

$$
\begin{aligned}
\delta E= & \int_{0}^{t_{f}} \int_{(\Omega)}\left[-S \frac{\partial \psi}{\partial t}-\nabla(T \nabla \psi)+\frac{\partial f}{\partial \phi}\right] \delta \phi \mathrm{d} \Omega \mathrm{d} t \\
& +\int_{0}^{t_{f}} \int_{(\Omega)}\left(\begin{array}{c}
\left(\frac{\partial f}{\partial p x_{m}}+(\nabla \psi \nabla \phi) \frac{\partial T}{\partial p x_{m}}\right) \delta p x_{m} \\
+\left(\frac{\partial f}{\partial p y_{m}}+(\nabla \psi \nabla \phi) \frac{\partial T}{\partial p y_{m}}\right) \delta p y_{m} \\
+\left(\frac{\partial f}{\partial T_{m}}+(\nabla \psi \nabla \phi) \frac{\partial T}{\partial T_{m}}\right) \delta T_{m}
\end{array}\right) \mathrm{d} \Omega \mathrm{d} t .
\end{aligned}
$$

Since $\psi(x, y, t)$ is the arbitrary function, it can be assumed as

$S \frac{\partial \psi}{\partial t}+\nabla \cdot(T \nabla \psi)-\frac{\partial f}{\partial \phi}=0$.

The final condition and boundary conditions are shown below.

$\psi\left(x, y, t_{f}\right)=0$,

$\left.\psi(x, y, t)\right|_{\left(\Gamma_{1}\right)}=0,\left.\quad T \frac{\partial \psi}{\partial n}\right|_{\left(\Gamma_{2}\right)}=0$.

Rearranging Eq. (16) with Eq. (17) and noting $\delta f / \delta p x_{m}=0$, the derivation of $\delta E / \delta p x_{m}$ can be obtained:

$\frac{\delta E}{\delta p x_{m}}=\int_{0}^{t_{f}} \int_{(\Omega)}\left((\nabla \psi \nabla \phi) \frac{\partial T}{\partial p x_{m}}\right) \mathrm{d} \Omega \mathrm{d} t$,

where $\frac{\delta p y_{m}}{\delta p x_{m}}=0$ and $\frac{\delta T_{m}}{\delta p x_{m}}=0$. Meanwhile, $\delta E / \delta p x_{m}$ and $\delta E /$ $\delta p x_{m}$ can be derived in the same way

$$
\begin{aligned}
& \frac{\delta E}{\delta p y_{m}}=\int_{0}^{t_{f}} \int_{(\Omega)}\left((\nabla \psi \nabla \phi) \frac{\partial T}{\partial p y_{m}}\right) \mathrm{d} \Omega \mathrm{d} t, \\
& \frac{\delta E}{\delta T_{m}}=\int_{0}^{t_{f}} \int_{(\Omega)}((\nabla \psi \nabla \phi)) \mathrm{d} \Omega \mathrm{d} t .
\end{aligned}
$$

We note in Eq. (22), " $\nabla \psi \nabla \phi "$ directly relates to the zonal area of the selected $T_{m}$ because the variation of $T_{m}$ does not change the zonal pattern but the value. However, the variation of variables $p x_{m}$ or $p y_{m}$ may change both the pattern and the value of the zonal structure.

Considering Eqs. (1) and (4), the spatial distribution of transmissivity can be regarded as the spatial distribution of hydraulic conductivity when the thickness of the aquifer is a constant or known. Thus, transmissivity $T_{m}$ can be replaced with hydraulic conductivity $K_{m}$. Consequently, $\delta E / \delta T_{m}$ can be replaced by $\delta E / \delta K_{m}$ in this study.

\subsection{Optimization framework for parameter structure identification}

For a given parameter dimension, we first use VT to automatically parameterize the spatial distribution of the hydraulic conductivity with variables $p x_{m}, p y_{m}$, and $K_{m}$. Then the ASM is applied to calculate the sensitivities of the objective function, $\delta E / \delta p x_{m}, \delta E / \delta p y_{m}$, and $\delta E / \delta K_{m}$, respectively. We compare the gradient of each parameter $\left(\delta E / \delta p x_{m}, \delta E / \delta p y_{m}\right.$, and $\left.\delta E / \delta K_{m}\right)$ to decide the direction for the next iteration. However, the exact step size of the gradient is not required because we only use the order of the gradient to decide the direction for the next iteration and apply the Tabu list and step size to determine the best neighbor solution which is also the moving mechanism of the Tabu search. In the traditional gradient-based algorithm, the search process always moves in the direction to improve the solution and has no chance to reach a worse solution. On the contrary, the gradient employed in this study helps the search process to reach both a better 
or worse solution which is totally different from the traditional gradient-based algorithm. We note from Eqs. (20)(22), to obtain all sensitivities of each parameter we only need to run the simulation model twice. One is to compute the head $\phi$ with the groundwater governing equation, while the other is to compute the arbitrary function $\psi$ with the adjoint equation. With all sensitivities of the objective function computed, an iteration of TS is carried out to identify which neighbor solution should be chosen as a new current solution for the next iteration. Note that if only TS is applied to determine the best neighbor solution, $(3 \times m \times 2)$ simulation runs are needed to calculate the objective function values of all neighbor solutions within one iteration, where $m$ represents the number of zones. In this way, the optimization efficiency of TS is significantly improved by the ASM and the number of simulation runs is reduced from $(3 \times m \times 2)$ to two. Thus, more feasible solutions can be explored with reduced computational effort. After the principles for stopping the TS search process are satisfied, the stopping criteria of parameter structure identification can be verified. The optimal parameter structure is determined once the stopping criteria of parameter structure identification are met. The flowchart of the integrated optimization algorithm is illustrated in Fig. 3.

\section{Numerical experiments}

To verify the proposed methodology, we conducted numerical experiments for two hypothetical cases: one with discrete hydraulic conductivity zones and another with a continuous hydraulic conductivity distribution. For Case 1 , we assume that the number of discrete hydraulic conductivity zones is known as prior information. There are three zonation scenarios tested. Then in Case 2, we further tested the efficiency and effectiveness of the proposed methodology for the purpose of identifying a continuous hydraulic conductivity distribution.

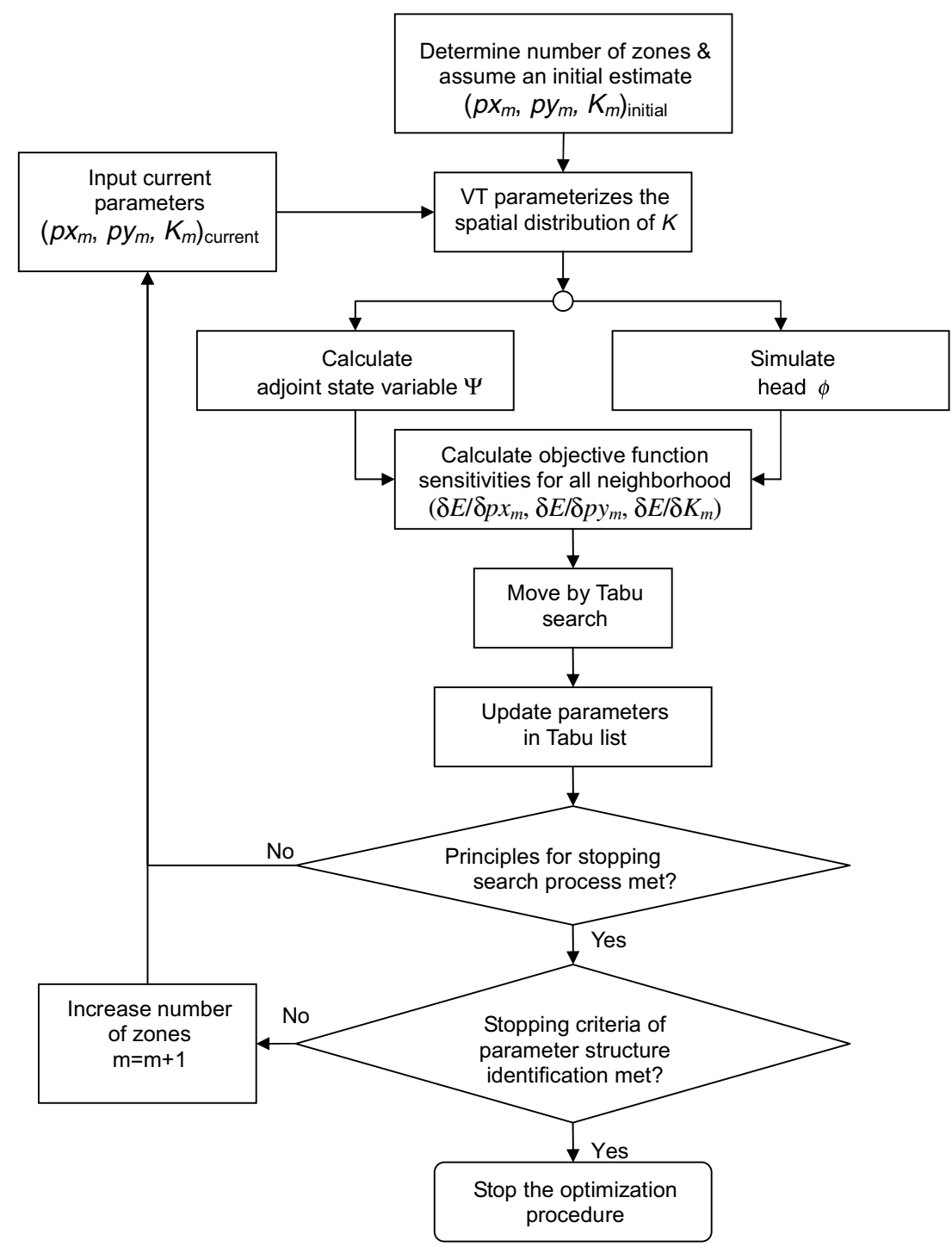

Fig. 3. Flowchart of the proposed integrated optimization algorithm. 


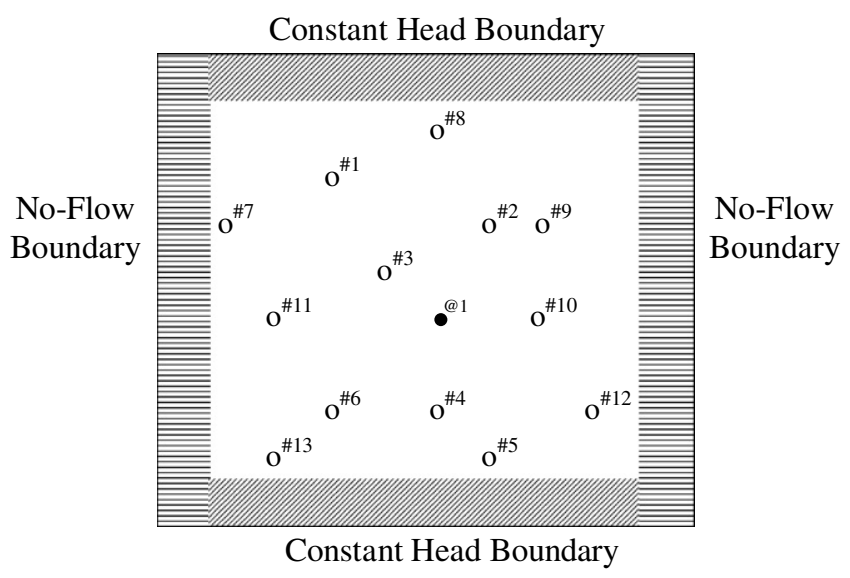

Fig. 4. The study area (\#1 to \#13 represent the observation wells, @1 represents the pumping well).

\subsection{Hypothetical aquifer}

Fig. 4 shows a hypothetical confined aquifer system. The dimension of the aquifer is $10 \times 10 \mathrm{~km}^{2}$ with a constant depth of $50 \mathrm{~m}$. The constant head boundary conditions are $10 \mathrm{~m}$ in the north and $30 \mathrm{~m}$ in the south. No flow boundary conditions apply to all other boundaries. Fig. 4 shows the locations of the 13 observation wells, \#1 to
Table 1

Parameter values used in the groundwater model

\begin{tabular}{clllll}
\hline Parameter & $\begin{array}{l}\text { Thickness } \\
\text { of aquifer } \\
(\mathrm{m})\end{array}$ & $\begin{array}{l}\text { Specific } \\
\text { storage } \\
(1 / \mathrm{m})\end{array}$ & $\begin{array}{l}\text { Simulation } \\
\text { period }(\mathrm{d})\end{array}$ & $\begin{array}{l}\text { Number } \\
\text { of } \\
\text { pumping } \\
\text { well }\end{array}$ & $\begin{array}{l}\text { Number of } \\
\text { observation } \\
\text { well }\end{array}$ \\
\hline $\begin{array}{c}\text { Design } \\
\text { value }\end{array}$ & 50 & 0.0001 & 10 & 1 & 13 \\
\hline
\end{tabular}

\#13. One pumping well is denoted as @ 1 in Fig. 4 and the pumping rate is constant with a value of $8640 \mathrm{~m}^{3} / \mathrm{d}$ from day 1 to day 5. The simulation period is $10 \mathrm{~d}$ and head observations are collected from these thirteen wells at the end of each day. In total, there are 130 head observations. These observations are subsequently corrupted with Gaussian noise of zero mean and standard deviation $\sigma_{h}=0.15 \mathrm{~m}$. The specific storage $\left(S_{\mathrm{s}}\right)$ is assumed homogenous and set at $0.0001(1 / m)$. Table 1 summarizes the design parameters.

\subsection{Formulation of the optimization model}

In Eq. (4), the residual error (RE) between observed and simulated heads is used as the objective function of the inverse problem. A mathematical model for the inverse problem can be formulated as
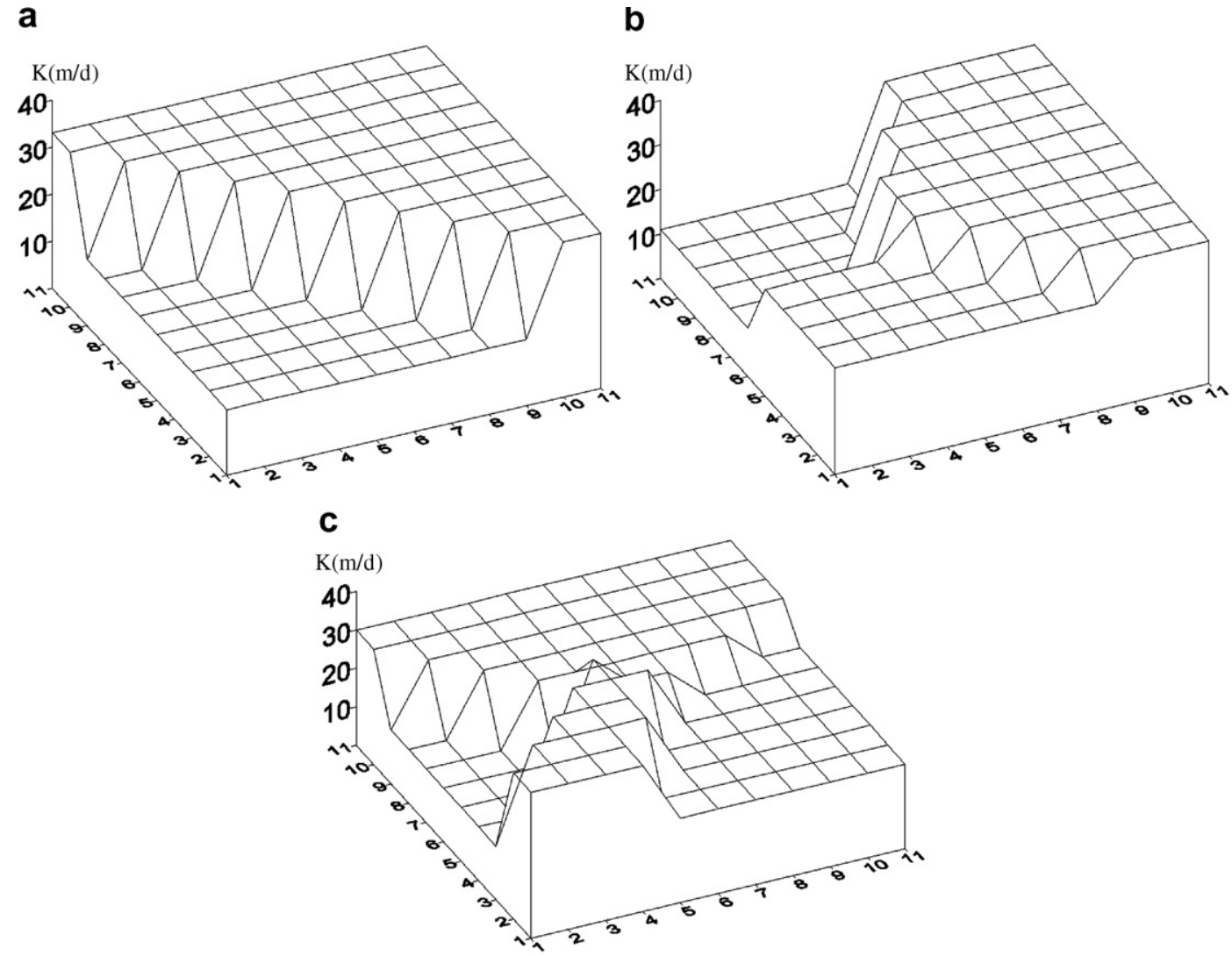

Fig. 5. The true spatial distributions of hydraulic conductivity $(\mathrm{m} / \mathrm{d})$ of the hypothetical field: (a) two zones (b) three zones (c) four zones ( $X$ and $Y$ axes represent the study area with $10 \times 10$ grids). 
$\operatorname{Min} E=\left[\frac{1}{N_{\text {well }} \times S P} \sum_{n=1}^{N_{\text {well }}} \sum_{t=1}^{S P}\left(\phi^{\operatorname{sim}}(n, t)-\phi^{\text {obs }}(n, t)\right)^{2}\right]^{1 / 2}$,

Zonation $\in\left[Z^{\mathrm{VT}}\left(p x_{i}, p y_{i}, K_{i}\right)\right]$,

$p x^{l} \leqslant p x_{i} \leqslant p x^{u}, \quad i=1, \ldots, m$,

$p y^{l} \leqslant p y_{i} \leqslant p y^{u}, \quad i=1, \ldots, m$,

$K^{l} \leqslant K_{i} \leqslant K^{u}, \quad i=1, \ldots, m$,

$\Omega=\left[\left(Z_{1}^{\mathrm{VT}}\right), \ldots,\left(Z_{m}^{\mathrm{VT}}\right)\right]$.

The parameter $N_{\text {well }}$ is the number of observed wells (including \#1 to \#13). SP is the simulation period. The $\phi^{\mathrm{sim}}(n, t)$ and $\phi^{\mathrm{obs}}(n, t)$ are the simulated and observed heads of well $n$ at time $t$. In Eq. (24), VT is applied as the zonation method to parameterize the spatial distribution of hydraulic conductivity. In Eqs. (25)-(27), the terms $p x^{l}, p x^{u}, p y^{l}, p y^{u}, K^{l}$, and $K^{u}$ represent the lower and upper bounds of the corresponding parameters. In this study, $p x^{l}$ and $p y^{l}$ are set at $1 \mathrm{~km}$, and $p x^{u}$ and $p y^{u}$ are set at $11 \mathrm{~km} . K^{l}$ and $K^{u}$ are set to be $1[\mathrm{~m} / \mathrm{d}]$ and $40[\mathrm{~m} / \mathrm{d}]$, respectively. In Eq. (27), $m$ is the number of zones and the spatial distribution of the hydraulic conductivity $(\Omega)$ is defined once the zonation $\left[Z^{\mathrm{VT}}\left(p x_{i}, p y_{i}, K_{i}\right)\right]$ is determined. Thus, the deci- sion variables of this optimization problem are $p x_{i}, p y_{i}$, $K_{i}, i=1, \ldots, m$.

\subsection{Case 1: Discrete hydraulic conductivity zone}

Fig. 5 shows the three zonation patterns used for generating the observations at the 13 observation well locations. Using only the corrupted observations, the inverse problem seeks to uncover (identify) the true spatial distributions. In this study we adopt PMWIN (Processing MODFLOW for Windows), developed by Chiang and Kinzelbach [20], to model the groundwater flow. We also use the software, Visual Basic (VB), to write the codes to perform the Tabu search and the integrated optimization. Additionally, we use the VB module to execute MODFLOW which also solves the adjoint state equation.

\subsubsection{Specification of $T S$}

As for the specification of TS, the neighborhood can be constructed after the step sizes of all decision variables are defined. In Case 1, the step sizes of $p x_{i}, p y_{i}$, and $K_{i}$ are fixed at $\Delta p x_{i}=1 \mathrm{~km}, \Delta p y_{i}=1 \mathrm{~km}$, and $\Delta K_{i}=1[\mathrm{~m} / \mathrm{d}]$. The movements then are defined to adjust each decision variable one step at a time. There are two directions for each
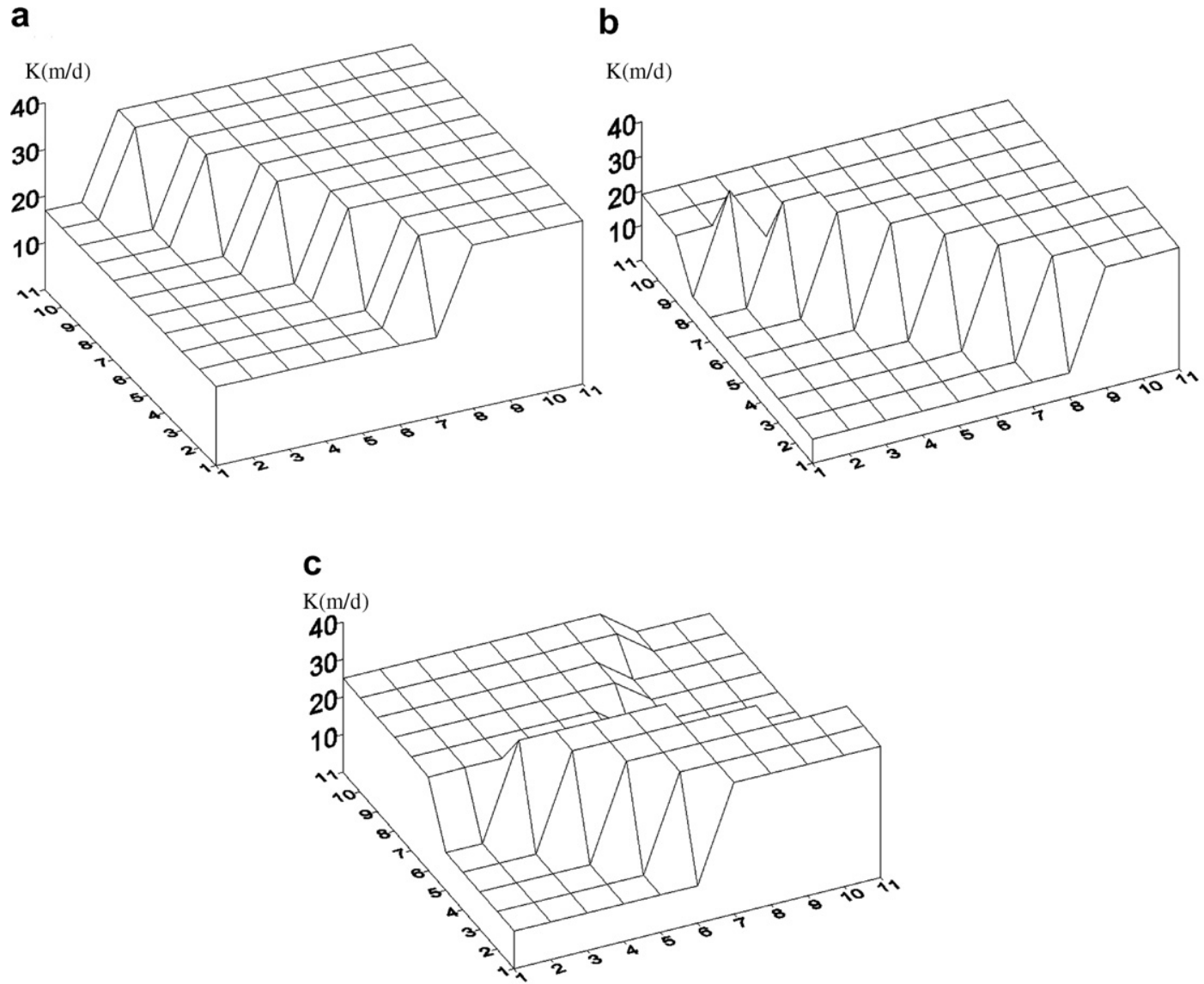

Fig. 6. The spatial distributions of hydraulic conductivity (m/d) of the initial solution: (a) two zones (b) three zones (c) four zones ( $X$ and $Y$ axes represent the study area with $10 \times 10$ grids). 
variable, reflecting a positive or negative step. Each zone has three parameters, and each parameter has two directions for modification. Thus the number of neighbor solutions is related to the number of zones. For example, if there are two zones, the six decision variables for $Z^{\mathrm{VT}}$ are $\left\{p x_{1}, p y_{1}, K_{1}, p x_{2}, p y_{2}, K_{2}\right\}$. Therefore, the number of neighbor solutions is $(3 \times 2 \times 2)$. Increasing the number of zones $i$ will result in more decision variables and also enlarge the neighborhood. The search process is stopped when the current solution satisfies the pre-defined residual error tolerance which is $0.15 \mathrm{~m}$, or the standard deviation of the observation errors or successive iterations do not improve the objective function value. The Tabu list needs to define recorded items and the length of the list. When any parameter of zone $i$ of the current solution is selected to be changed for a movement, the three parameters of the $i$ th zone are recorded in the Tabu list. For example, if $p y_{1}$ is selected to be changed, $p x_{1}, p y_{1}, K_{1}$ are all recorded. As a part of the Tabu search, we also use some aspiration criteria for keeping the search process from premature termination. For example, a movement is allowed when the value of some parameters of a neighbor solution is tabued but the neighbor solution has a better objective function value than the recorded best solution. And all the tabued memories are erased as long as all neighbor solutions are tabued.
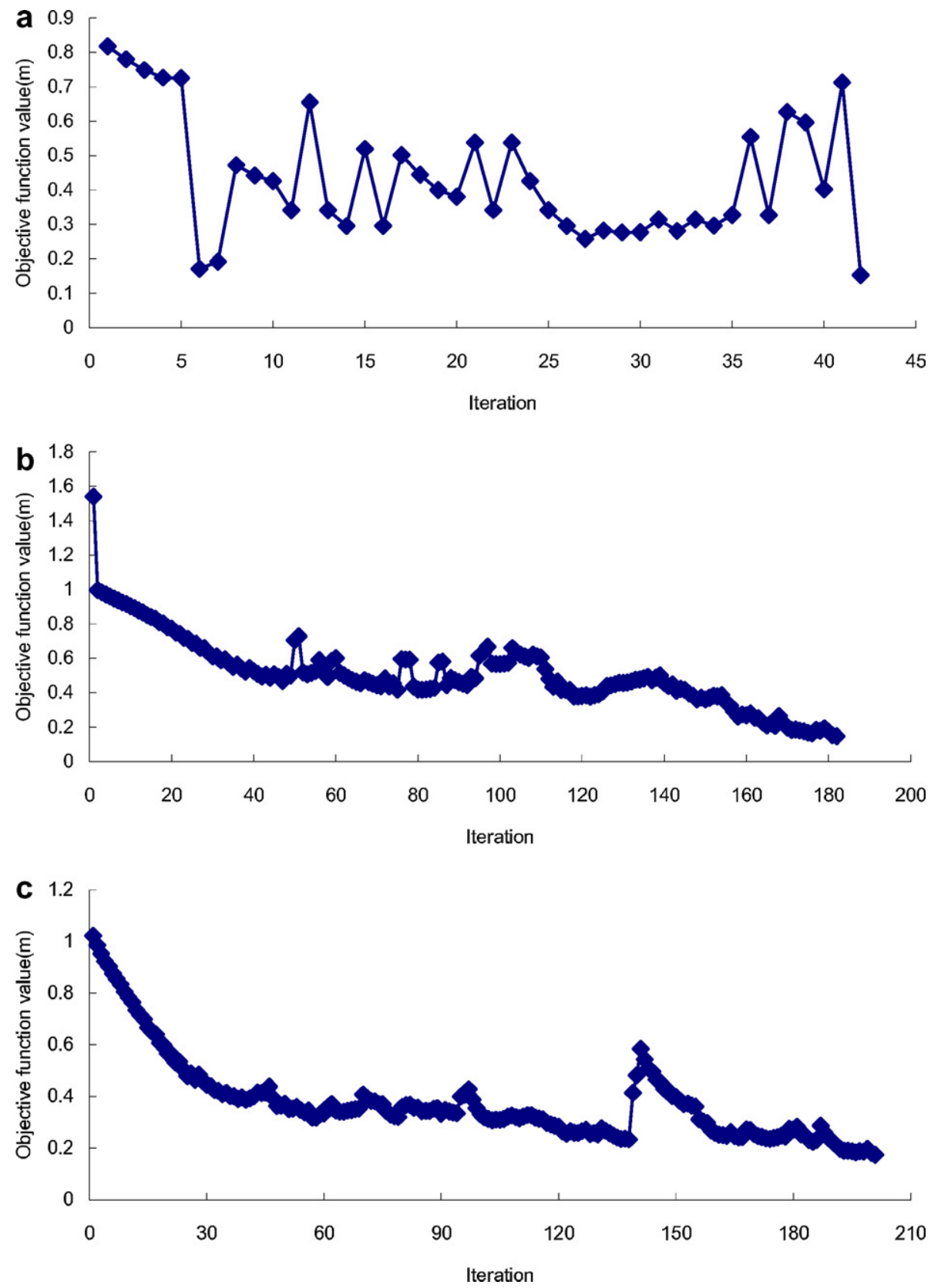

Fig. 7. Tracking the objective function values with each iteration: (a) two zones (b) three zones (c) four zones. 
a

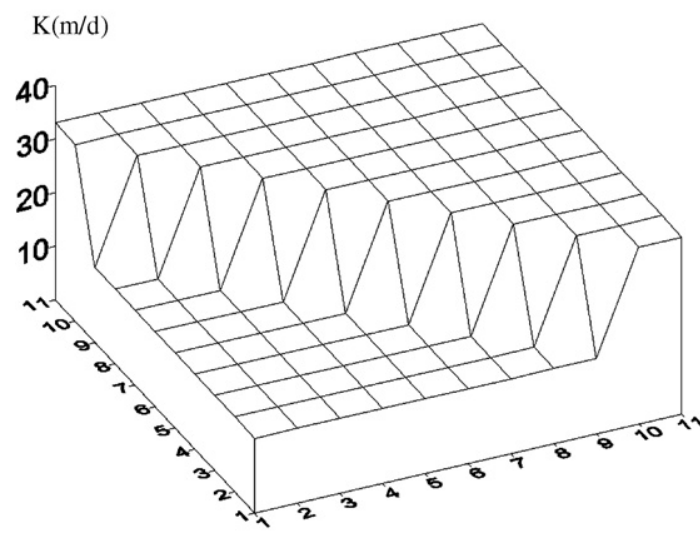

b

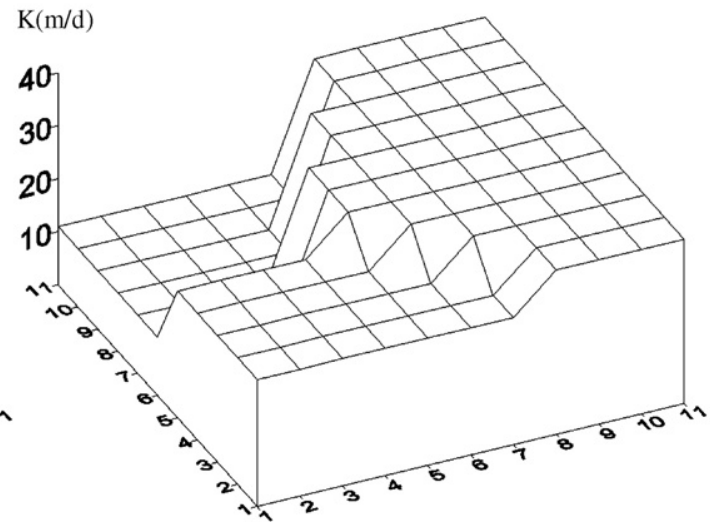

C

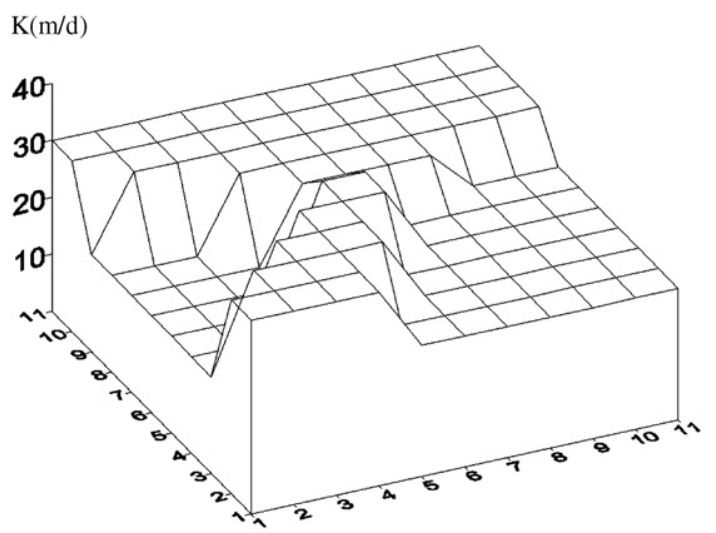

Fig. 8. The spatial distributions of hydraulic conductivity $(\mathrm{m} / \mathrm{d})$ of the optimal solution: (a) two zones (b) three zones (c) four zones $(X$ and $Y$ axes represent the study area with $10 \times 10$ grids).

\subsubsection{Parameter identification results}

Fig. 6 shows the spatial distribution of hydraulic conductivity with randomly assigned $K$ values as the initial solutions for the two zone, three zone, and four zone scenarios, respectively. Comparing the true hydraulic conductivity in Fig. 5 with the initial solution in Fig. 6, the two spatial distributions are notably different both in shape and value. Then TS is allied with ASM to optimize these parameters $\left\{p x_{i}, p y_{i}, K_{i}\right\}$ for the three respective zonation scenarios with the objective of minimizing the residual error. Fig. 7 shows the objective function value for each iteration. The optimal solution is identified at the 42th iteration for the two zone case, the 182th iteration for the three zone case, and the 201th iteration for the four zone case. With the increasing number of zones, the required iterations to reach the optimum are larger due to the more complicated parameter structures Fig. 8 illustrates the optimal spatial distributions of hydraulic conductivity of two zones, three zones, and four zones. Table 2 shows the results in detail. As can be seen from Table 2, the optimal values of hydraulic conductivity are not exactly the same with the true values due to observation errors but the patterns are very similar. The search processes of two zones and four zones are stopped when there are no improve-
Table 2

Results of optimal spatial distributions of hydraulic conductivity

\begin{tabular}{|c|c|c|c|c|c|c|}
\hline & \multicolumn{2}{|c|}{ Two zones } & \multicolumn{2}{|c|}{ Three zones } & \multicolumn{2}{|c|}{ Four zones } \\
\hline & True & Optimal & True & Optimal & True & Optimal \\
\hline $\mathrm{K} 1(\mathrm{~m} / \mathrm{d})$ & 14 & 14 & 11 & 11 & 14 & 17 \\
\hline $\mathrm{K} 2(\mathrm{~m} / \mathrm{d})$ & 33 & 33 & 24 & 24 & 22 & 23 \\
\hline $\mathrm{K} 3(\mathrm{~m} / \mathrm{d})$ & - & - & 32 & 31 & 30 & 30 \\
\hline $\mathrm{K} 4(\mathrm{~m} / \mathrm{d})$ & - & - & - & - & 38 & 34 \\
\hline RE (m) & - & 0.153 & - & 0.147 & - & 0.174 \\
\hline
\end{tabular}

ments in residual error after successive iterations. The larger residual error with four zones implies that the observed heads with observation error of $\sigma_{h}=0.15 \mathrm{~m}$ may not be sufficient to identify a four zone structure. Identifying a more complex parameter structure requires more data of good quality.

To demonstrate the advantage of embedding ASM in the Tabu search, we solve the same problems only with TS. Comparisons of these two approaches are shown in Table 3. The proposed TS allied with ASM requires 84, 364, and 404 MODFLOW simulation runs (each iteration needs 2 runs) to reach the optimum for the two zone, three zone, and four zone cases, respectively. Using TS alone requires 432, 1872, and 5592 MODFLOW simulation runs 
Table 3

Comparisons between TS and TS allied with ASM

\begin{tabular}{|c|c|c|c|c|c|c|c|}
\hline & & $\begin{array}{l}\text { Initial } \\
\mathrm{RE}(\mathrm{m})\end{array}$ & $\begin{array}{l}\text { Optimal } \\
\text { RE (m) }\end{array}$ & Length of Tabu list & Iterations & $\begin{array}{l}\text { Number of MODFLOW runs } \\
\text { needed in one iteration }\end{array}$ & $\begin{array}{l}\text { Total number of } \\
\text { MODFLOW runs }\end{array}$ \\
\hline \multirow[t]{2}{*}{ Two zones } & TS with ASM & 0.81 & 0.153 & 8 & 42 & 2 & 84 \\
\hline & $\mathrm{TS}$ & 0.81 & 0.153 & 6 & 36 & 12 & 432 \\
\hline \multirow[t]{2}{*}{ Three zones } & TS with ASM & 1.54 & 0.147 & 14 & 182 & 2 & 364 \\
\hline & $\mathrm{TS}$ & 1.54 & 0.145 & 12 & 104 & 18 & 1872 \\
\hline \multirow[t]{2}{*}{ Four zones } & TS with ASM & 1.02 & 0.174 & 40 & 201 & 2 & 404 \\
\hline & TS & 1.02 & 0.161 & 30 & 233 & 24 & 5592 \\
\hline
\end{tabular}

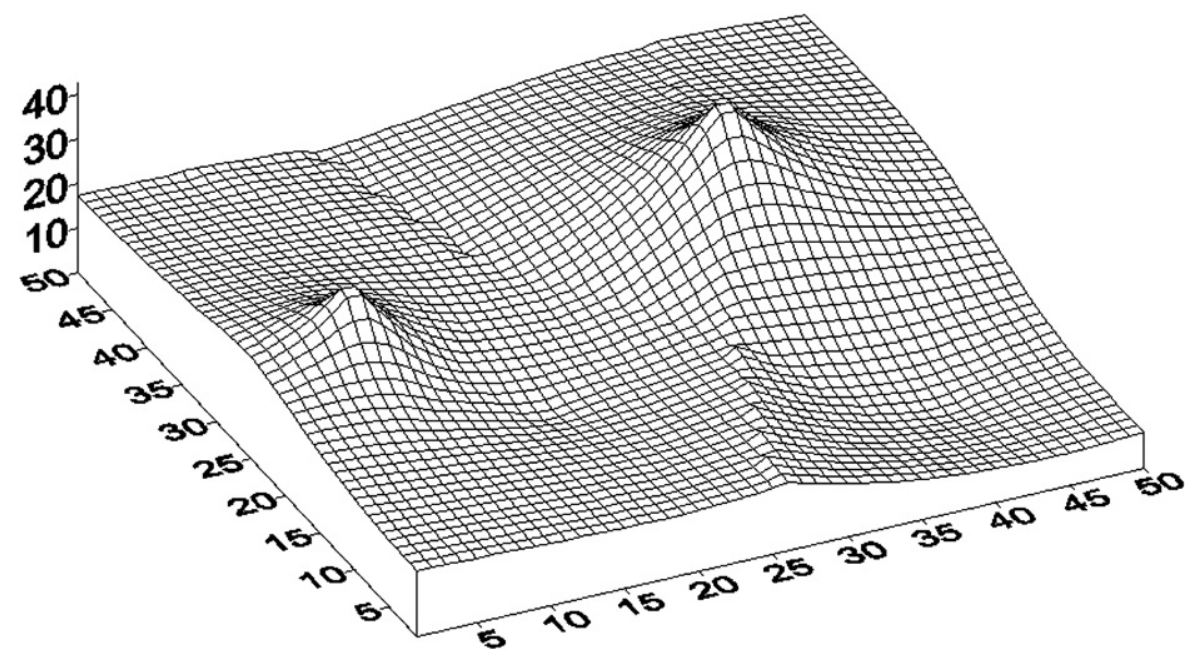

Fig. 9. The continuous spatial distributions of hydraulic conductivity $(\mathrm{m} / \mathrm{d})$ of the hypothetical field ( $X$ and $Y$ axes represent the study area with lengths of $10 \mathrm{~km})$.

(each iteration needs 12,18 , and 24 runs, respectively) to identify the optimal spatial distribution of hydraulic conductivity. It is evident that both methods have the ability to reach near global optima, but when TS is allied with ASM, the number of simulation runs is significantly reduced. This is particularly true for higher parameter dimensions.

Since the search process of the proposed algorithm reaches cycles with the same lengths of Tabu list of the TS, a longer length of Tabu list is assigned as shown in Table 3. Basically, the search process of TS is deterministic as long as an initial solution and the length of the Tabu list are fixed. However, the TS allied with ASM computes the sensitivities - the tangent slope - to determine a movement. TS alone has to calculate the neighbor objective function value to decide the best neighbor solution. Thus, the search process and the number of iterations of both methods are not identical. The TS allied with ASM may require more iterations, but with much less effort to evaluate neighbor solutions in searching for the direction toward local optimums, while TS alone may require fewer iterations, but with much more effort to evaluate neighbor solutions. However, both methods can find satisfying results but with different efficiency. Thus, this study further proposes to apply TS alone with larger steps of movement. Then, TS allied with ASM will be applied to refine the solution with finer steps of movement. Finally, a coarse-fine grid technique which is analogous to the quality memory of TS is used in Case 2 to identify a continuous hydraulic conductivity distribution.

\subsection{Case 2: Continuous hydraulic conductivity distribution}

We use the parameterization method by Tan et al. [21] to generate the continuous hydraulic conductivity field as shown in Fig. 9. We then use this field to generate observations. Again, the generated observations are corrupted with Gaussian noise with different standard deviations. Using only the corrupted observations, we seek to identify the best parameter structure to capture the spatial variation of the heterogeneous field.

\subsubsection{Specification of TS}

As for the specification of TS, there is only one difference between Case 1 and Case 2. Rather than using a fixed step size as in Case 1, Case 2 adopts a coarse-fine grid technique to adjust the step size. A coarse grid with $\Delta p x_{i}=2 \mathrm{~km}, \Delta p y_{i}=2 \mathrm{~km}$, and $\Delta K_{i}=4[\mathrm{~m} / \mathrm{d}]$ is utilized to approximate the optimal basis point locations and the associated values. Faster convergence is expected with the 
a

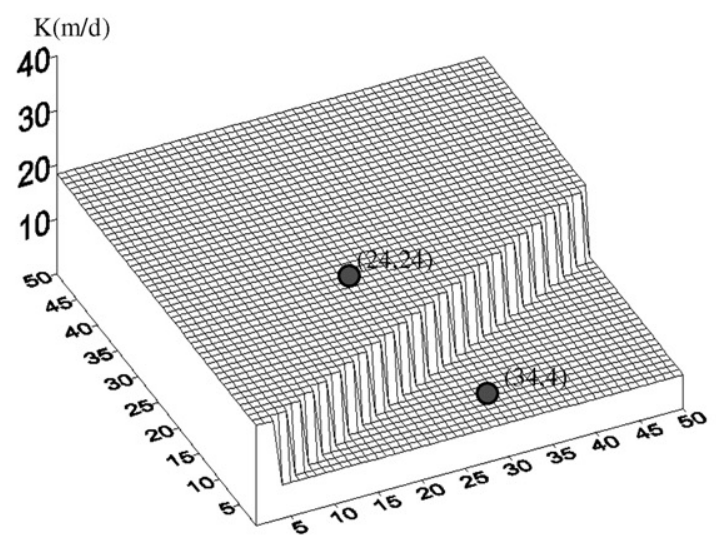

C

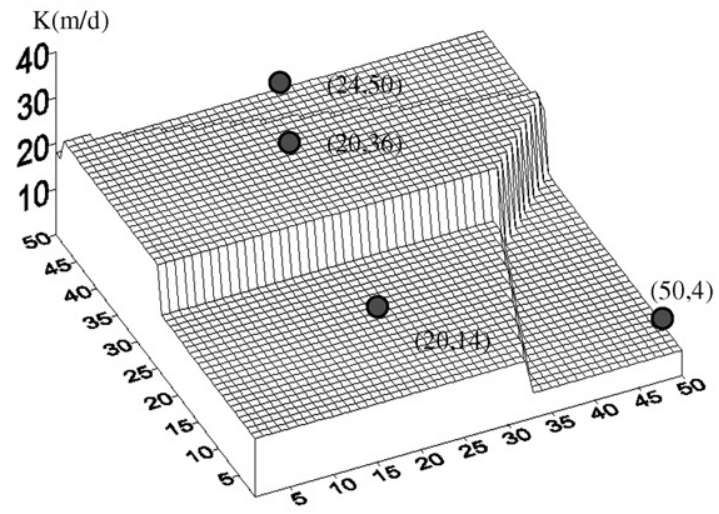

b

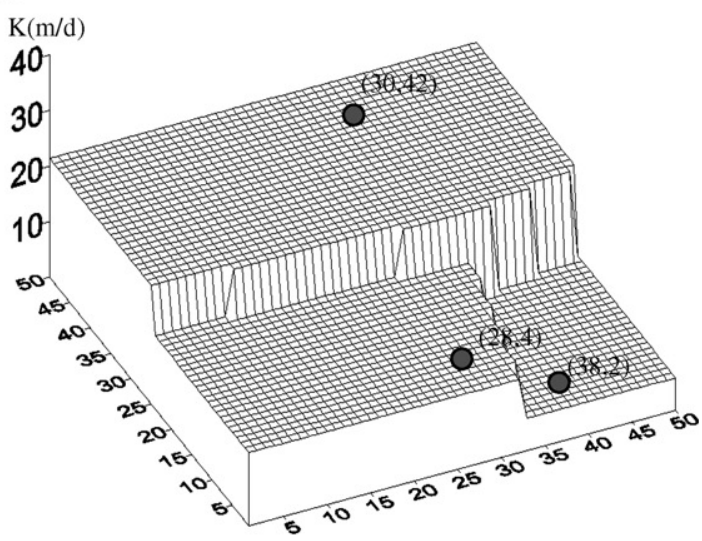

d

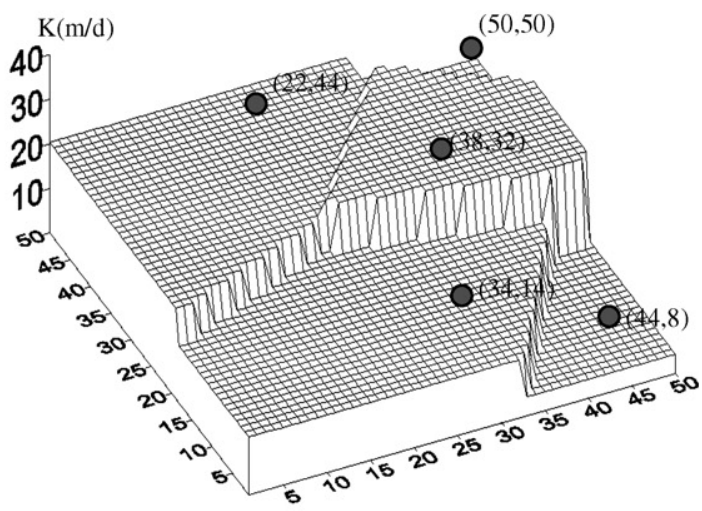

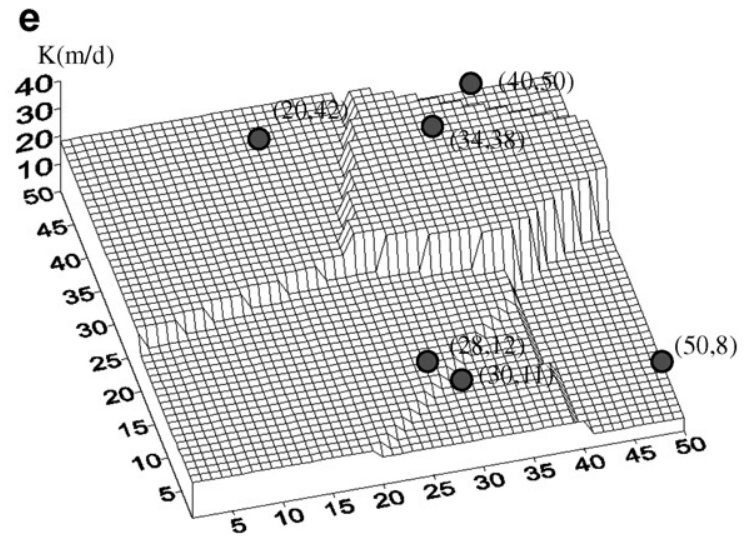

Fig. 10. The optimal spatial distributions of hydraulic conductivity (m/d): (a) two zones (b) three zones (c) four zones (d) five zones (e) six zones.

coarse grid. The optimal solution from the coarse grid is then chosen as the initial solution for the fine grid with $\Delta p x_{i}=400 \mathrm{~m}, \Delta p y_{i}=400 \mathrm{~m}$, and $\Delta K_{i}=0.2[\mathrm{~m} / \mathrm{d}]$. The optimization procedure with the coarse-fine grid technique starts from two zones and continues to increase the number of zones. For each level of complexity, we calculate the residual error, the parameter uncertainty error and $A I C_{c}$.

\subsubsection{Parameter identification results}

Fig. 10 shows the optimal spatial distribution of hydraulic conductivity of two zones, three zones, four zones, five zones, and six zones. Compared to the true hydraulic con- ductivity distribution in Fig. 9, the optimized parameter patterns after four zones start to capture the variation of the true distribution. Table 4 shows the optimization results. As can be seen from the results and Fig. 11, the residual error decreases not only from coarse grid to fine grid but also from two zones to six zones. It is obvious that with more zones the fit improves as far as the residual error is concerned. Although the residual error decreases as the number of zones increases, caution must be exercised so that over-parameterization is avoided. In many instances, a model with fewer parameters may have greater sensitivity and less uncertainty. Therefore, we examine two other 
Table 4

Results of the integrated optimization algorithm with $\sigma_{h}=0.15 \mathrm{~m}$

\begin{tabular}{|c|c|c|c|c|c|c|c|}
\hline \multirow[t]{2}{*}{ Structure } & \multicolumn{2}{|c|}{ TS with coarse grid } & \multicolumn{5}{|c|}{ TS with ASM and fine grid } \\
\hline & $K_{i}(\mathrm{~m} / \mathrm{d})$ & $\mathrm{RE}(\mathrm{m})$ & $K_{i}(\mathrm{~m} / \mathrm{d})$ & $\mathrm{RE}(\mathrm{m})$ & $\operatorname{tr}(\mathrm{Cov}) \times 10^{4}$ & $A I C_{c}$ & $A I C$ \\
\hline Two zones (6 Param.) & $\begin{array}{r}20 \\
8\end{array}$ & 0.632 & $\begin{array}{r}18.4 \\
6.2\end{array}$ & 0.58 & 3.53 & -46.59 & -49.51 \\
\hline Three zones (9 Param.) & $\begin{array}{r}28 \\
4 \\
12\end{array}$ & 0.448 & $\begin{array}{r}21.4 \\
5.8 \\
13.2\end{array}$ & 0.281 & 4.39 & -121.49 & -125.34 \\
\hline Four zones (12 Param.) & $\begin{array}{r}20 \\
24 \\
4 \\
12\end{array}$ & 0.289 & $\begin{array}{r}18 \\
22.8 \\
5.4 \\
12.8\end{array}$ & 0.232 & 7.15 & -136.32 & -141.46 \\
\hline Five zones (15 Param.) & $\begin{array}{r}16 \\
12 \\
32 \\
4 \\
12\end{array}$ & 0.264 & $\begin{array}{c}11.8 \\
20.4 \\
23.4 \\
4.2 \\
13\end{array}$ & 0.215 & 25.8 & -136.75 & -143.57 \\
\hline Six zones (18 Param.) & $\begin{array}{r}24 \\
12 \\
20 \\
4 \\
20 \\
12\end{array}$ & 0.257 & $\begin{array}{c}25.4 \\
12.8 \\
18.4 \\
4.8 \\
10.4 \\
10\end{array}$ & 0.209 & - & -131.31 & -140.22 \\
\hline
\end{tabular}

indices; that is, the parameter uncertainty error and $A I C_{c}$. From Table 4, for $\sigma_{h}=0.15 \mathrm{~m}$ the optimal number of zones is four. Parameter dimension higher than four results in a drastic increase in parameter uncertainty error. The parameter uncertainty error of five zones is almost $261 \%$ greater than the parameter uncertainty error of four zones. We note that the $A I C_{c}$ value of five zones is only $0.32 \%$ less than the $A I C_{c}$ value of four zones. This implies that the $A I C_{c}$ value approaches a minimum around four zones since there is no significant improvement in $A I C_{c}$ beyond four zones. For comparison purposes, we also presented the $A I C$ values in Table 4. For this particular example, the $A I C$ and $A I C_{c}$ values show consistent results. We also note that in Table 4 the residual error is further improved from coarse grid to fine grid. Both the basis point locations and the hydraulic conductivity values are changed to produce a better fit. However, most of the changes are associated with the hydraulic conductivity rather than the basis point locations. Tsai and Yeh [22] also showed that the sensitivity of basis point location is lower than that of the hydraulic conductivity.

We also apply the proposed methodology to the same set of observations but corrupted with different observation errors of $\sigma_{h}=0.45 \mathrm{~m}$ and $\sigma_{h}=0.03 \mathrm{~m}$. The results are shown in Tables 5 and 6. Generally, observations with less errors provide better information for parameter structure identification, which in turn allow for a higher parameter dimension. Again, the residual error continues to decrease with increasing parameter dimension, but the parameter uncertainty error and $A I C_{c}$ worsen. The results in Tables 5 and 6 suggest that the optimal numbers of zones are three with a larger observation error and five with a smaller

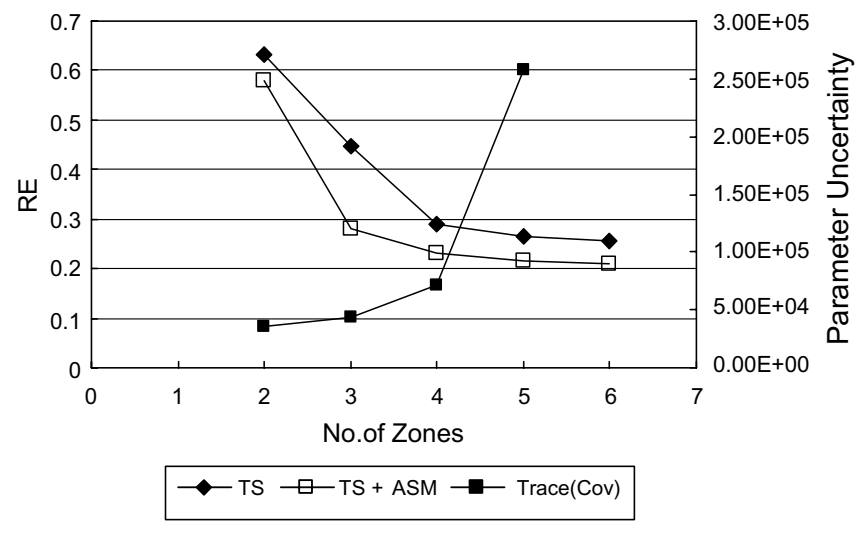

Fig. 11. Parameter dimension with residual error and parameter uncertainty.

observation error. These results are also consistent with the results when $\sigma_{h}=0.15 \mathrm{~m}$.

\section{Conclusions}

In this study, we have proposed an integrated optimization algorithm that combines TS with the ASM. Numerical experiments have demonstrated that this approach significantly reduces the number of simulation runs when compared to the traditional TS. The proposed methodology is particularly efficient when the number of parameters is large. Traditional TS requires evaluating all neighbor solutions in one iteration, whereas TS allied with the ASM only requires two simulation runs regardless of the parameter dimension. 
Table 5

Results of the integrated optimization algorithm with $\sigma_{h}=0.45 \mathrm{~m}$

\begin{tabular}{|c|c|c|c|c|c|c|}
\hline \multirow[t]{2}{*}{ Structure } & \multicolumn{2}{|c|}{ TS with coarse grid } & \multicolumn{4}{|c|}{ TS with ASM and fine grid } \\
\hline & $K_{i}(\mathrm{~m} / \mathrm{d})$ & $\mathrm{RE}(\mathrm{m})$ & $K_{i}(\mathrm{~m} / \mathrm{d})$ & $\mathrm{RE}(\mathrm{m})$ & $\operatorname{tr}(\mathrm{Cov}) \times 10^{4}$ & $A I C_{c}$ \\
\hline Two zones (6 Param.) & $\begin{array}{r}20 \\
8\end{array}$ & 0.722 & $\begin{array}{r}18.8 \\
6.8\end{array}$ & 0.672 & 3.65 & -29.97 \\
\hline Three zones (9 Param.) & $\begin{array}{r}20 \\
8 \\
12\end{array}$ & 0.58 & $\begin{array}{r}20.8 \\
4.6 \\
13.4\end{array}$ & 0.44 & 4.61 & -70.85 \\
\hline
\end{tabular}

Table 6

Results of the integrated optimization algorithm with $\sigma_{h}=0.03 \mathrm{~m}$

\begin{tabular}{|c|c|c|c|c|c|c|}
\hline \multirow[t]{2}{*}{ Structure } & \multicolumn{2}{|c|}{ TS with coarse grid } & \multicolumn{4}{|c|}{ TS with ASM and fine grid } \\
\hline & $K_{i}(\mathrm{~m} / \mathrm{d})$ & $\mathrm{RE}(\mathrm{m})$ & $K_{i}(\mathrm{~m} / \mathrm{d})$ & $\mathrm{RE}(\mathrm{m})$ & $\operatorname{tr}(\mathrm{Cov}) \times 10^{4}$ & $A I C_{c}$ \\
\hline Two zones (6 Param.) & $\begin{array}{r}20 \\
8\end{array}$ & 0.602 & $\begin{array}{r}19.4 \\
6.6\end{array}$ & 0.55 & 3.91 & -52.59 \\
\hline Three zones (9 Param.) & $\begin{array}{r}4 \\
12 \\
20\end{array}$ & 0.309 & $\begin{array}{r}4.2 \\
12.8 \\
20.8\end{array}$ & 0.241 & 4.44 & -138.83 \\
\hline Four zones (12 Param.) & $\begin{array}{r}24 \\
20 \\
4 \\
12\end{array}$ & 0.24 & $\begin{array}{c}19 \\
22.8 \\
6 \\
12.6\end{array}$ & 0.174 & 28.25 & -168.32 \\
\hline Five zones (15 Param.) & $\begin{array}{r}20 \\
24 \\
20 \\
4 \\
12\end{array}$ & 0.21 & $\begin{array}{c}13 \\
23.8 \\
20.2 \\
5.6 \\
13\end{array}$ & 0.124 & 32.3 & -198.9 \\
\hline Six zones (18 Param.) & $\begin{array}{r}40 \\
4 \\
20 \\
24 \\
12 \\
12\end{array}$ & 0.198 & $\begin{array}{c}24 \\
4.6 \\
19.4 \\
6.8 \\
8.6 \\
13\end{array}$ & 0.122 & 48.92 & -192.64 \\
\hline
\end{tabular}

We used VT as the zonation method in this study to parameterize the spatial distribution of hydraulic conductivity. There is no doubt that VT has some limitations for example, it can only delineate a straight boundary. However, VT still remains a powerful tool for automatic parameterization of a heterogeneous field. The proposed optimization algorithm was verified to reasonably identify both discrete hydraulic conductivity zones and the continuous hydraulic conductivity distribution.

We added a coarse-fine grid technique to the proposed algorithm to further improve computational efficiency. A coarse grid search was conducted to reduce the feasible region. The optimal solution obtained from the coarse grid search was used as the initial estimate for the fine grid search.

The ability to identify parameter structure complexity is directly related to data quantity as well as quality. Better observations afford more information to identify a more complex parameter structure. The ASM is applicable for deriving the sensitivity of any other parameters such as the storage coefficient, the pumping history, and the release history in groundwater flow and mass transportation. Thus, the proposed integrated optimization algorithm can be extended to other studies. Moreover, different parameterization methods can be used to replace the VT and increase spatial variability.

\section{References}

[1] Yeh WW-G. Review of parameter identification procedure in groundwater hydrology: the inverse problem. Water Resource Res 1986;22(2):95-108.

[2] Sykes JF, Wilson JL, Andrews RW. Sensitivity analysis for steady state groundwater flow using adjoint operators. Water Resource Res 1985;21(3):359-71. 
[3] Sun NZ, Yeh WW-G. Coupled inverse problem in groundwater modeling, 1, sensitivity analysis and parameter identification. Water Resource Res 1990;26(10):2507-25.

[4] Zheng C, Wang P. Parameter structure identification using tabu search and simulated annealing. Adv Water Resource 1996;19(4):215-24.

[5] Tsai FT-C, Sun NZ, Yeh WW-G. A combinatorial optimization scheme for parameter structure identification in ground water modeling. Ground Water 2003;41(2):156-69.

[6] Tung CP, Chou CA. Pattern classification using tabu search to identify the spatial distribution of groundwater pumping. Hydrogeol J 2004;12:488-96.

[7] Tung CP, Tan CC. An optimal procedure for identifying parameter structure and application to a confined aquifer. Environ Geol 2005;47:1062-71.

[8] Doherty J. Ground water model calibration using pilot points and regularization. Ground Water 2003;41(2):170-7.

[9] Sun NZ, Yang S, Yeh WW-G. A proposed stepwise regression method for model structure identification. Water Resource Res 1998;34(10):2561-72.

[10] Harbaugh AW, McDonald MG. User's documentation for MODFLOW 96 an update to the U.S. Geological Survey modular finitedifference groundwater flow model. US Geol Surv Open File; 1996.

[11] Yeh WW-G, Yoon YS. Aquifer parameter identification with optimum dimension in parameterization. Water Resource Res 1981;17(3):664-72.

[12] Akaike H. A new look at the statistical model identification. IEEE Trans Automat Control 1974;19:716-23.

[13] Hurvich CM, Tsai CL. Regression and time series model selection in small samples. Biometrika 1989;76:297-307.
[14] Dirichlet GL. Uber die Reduction der Positeven quadratischen Formen mit drei unbestimmten ganzen Zahlen. Journal of fur die Reine und Angewandte Mathematik 1850;40:209-27.

[15] Voronoi G. Nouvelles applications des parameters continues a la theorie des formes quadratiques. Deuxieme Memoire: Recherches sur les parallelloedres primitives. Journal fur die Reine und Angewandte Mathematik 1908;134:198-287.

[16] Okabe A, Boots B, Sugihara K. Spatial tessellations: concepts and applications of Voronoi diagrams. 1st ed. Chichester: John Wiley \& Sons; 1992.

[17] Glover F, Laguna M. Tabu search. Boston, MA, USA: Kluwer Academic Publishers; 1997.

[18] Sun NZ. Inverse problems in groundwater modeling. Norwell, MA: Kluwer Academic; 1994.

[19] Michalak AM, Kitanidis PK. Estimation of historical groundwater contaminant distribution using the adjoint state method applied to geostatistical inverse modeling. Water Resource Res 2004;40:W08302. doi:10.1029/2004WR003214.

[20] Chiang WH, Kinzelbach W. 3D-groundwater modeling with PMWIN: a simulation system for modeling groundwater flow and pollution. 1st ed. 2001. Corr. 3rd printing, vol. XIV. Springer; 2003. p. 346.

[21] Tan CC, Tung CP, Tsai FT-C. Applying zonation methods and Tabu search to improve the groundwater modeling. JAWRA, in press.

[22] Tsai FT-C, Yeh WW-G. Characterization and identification of aquifer heterogeneity with generalized parameterization and Bayesian estimation. Water Resource Res 2004;40:W10102. doi:10.1029/ 2003WR002893. 